\title{
Alterações estruturais e comportamento compressivo de um Latossolo Vermelho distrófico argiloso sob diferentes sistemas de uso e manejo(1)
}

\author{
Geraldo César de Oliveira ${ }^{(2)}$, Moacir de Sousa Dias Junior ${ }^{(3)}$, Dimas Vital Siqueira Resck ${ }^{(4)}$ e Nilton Curi( ${ }^{(3)}$
}

Resumo - Uma das principais consequiências do manejo inadequado do solo é a compactação, que leva à perda da sua sustentabilidade e à redução da produtividade. O objetivo deste estudo foi avaliar as alterações estruturais e o comportamento compressivo de um Latossolo Vermelho distrófico sob Cerrado $(\mathrm{C})$, plantio direto (PD) e preparo com arado de discos (AD), após duas décadas de uso e manejo. Nas profundidades de 0-5 cm e 20-30 cm coletaram-se amostras indeformadas para medir a pressão de preconsolidação, densidade do solo e a sua porosidade, e amostras deformadas para a caracterização física e química do solo. A densidade do solo variou na seguinte ordem: $\mathrm{PD}=\mathrm{AD}>\mathrm{C}(0-5 \mathrm{~cm})$ e $\mathrm{AD}>\mathrm{PD}>\mathrm{C}(20-30 \mathrm{~cm})$. Os dados de matéria orgânica mostraram que $\mathrm{C}=\mathrm{PD}>\mathrm{AD}(0-5 \mathrm{~cm}) \mathrm{e}$ $\mathrm{C}=\mathrm{PD}=\mathrm{AD}(20-30 \mathrm{~cm})$, demonstrando a capacidade de incremento da matéria orgânica pelo PD. A pressão de preconsolidação variou na seguinte ordem: $\mathrm{PD}=\mathrm{C}>\mathrm{AD}(0-5 \mathrm{~cm}$; tensão de $-1.500 \mathrm{kPa})$, e $\mathrm{AD}>\mathrm{C}=\mathrm{PD}(20-30 \mathrm{~cm}$; tensão de $-1.500 \mathrm{kPa})$.

Termos para indexação: plantio direto, arado de disco, densidade do solo, porosidade do solo, pressão.

\section{Structural changes and compressive behavior of a dystrophic clayey Red Latosol under different use and management systems}

\begin{abstract}
One of the consequences of inadequate soil management is its compaction that causes the loss of its sustainability and reduction of its productivity. The objective of this study was to evaluate the structural changes and the compressive behavior of a Latosol (Oxisol) under native vegetation, "Cerrado" (C), no-till (NT) and conventional till (CT) system, after two decades of use. The undisturbed soil samples were used to characterize the soil bulk density, the porous space and to perform the uniaxial compression test. The disturbed soil samples were used for soil physical and chemical characterization analyses in the analyzed depth. The bulk density varied in the following order: $\mathrm{NT}=\mathrm{CT}>\mathrm{C}(0$ to $5 \mathrm{~cm})$ and $\mathrm{CT}>\mathrm{NT}>\mathrm{C}(20$ to $30 \mathrm{~cm})$. Organic matter data showed that in the $\mathrm{C}=\mathrm{NT}>\mathrm{CT}(0$ to $5 \mathrm{~cm})$ and $\mathrm{C}=\mathrm{NT}=\mathrm{CT}(20$ to $30 \mathrm{~cm})$, showing the NT capacity of increasing organic matter. The preconsolidation pressure changed in the following order: $\mathrm{NT}=\mathrm{C}>\mathrm{CT}(0$ to $5 \mathrm{~cm}$; tension $-1,500 \mathrm{kPa})$, and $\mathrm{CT}>\mathrm{C}=\mathrm{NT}(20$ to $30 \mathrm{~cm}$; tension $-1,500 \mathrm{kPa})$.
\end{abstract}

Index terms: direct sowing, disc ploughs, soil density, soil pore system, pressure.

(1) Aceito para publicação em 24 de setembro de 2002 .

Parte da tese de doutorado do primeiro autor apresentada à Universidade Federal de Lavras (Ufla), Lavras, MG

Projeto parcialmente financiado pelo $\mathrm{CNPq}$.

(2) Universidade Federal de Goiás, Escola de Agronomia, Caixa Postal 131, CEP 74001-970 Goiânia, GO. Bolsista da Capes. E-mail: gcesar@agro.ufg.br

(3) Ufla, Dep. de Ciência do Solo, Caixa Postal 37, CEP 37200-000 Lavras, MG. Bolsista do CNPq. E-mail: msouzadj@ufla.br,niltcuri@ufla.br

(4) Embrapa-Centro de Pesquisa Agropecuária dos Cerrados, Caixa Postal 08223, CEP 73301-970 Planaltina, DF. Bolsista do CNPq. E-mail: dvsresck@cpac.embrapa.br

\section{Introdução}

A busca por alternativas tecnológicas que possibilitem o uso racional do solo tem sido a tônica das discussões em torno do tema manejo correto do solo para uma agricultura sustentável.

Dos componentes do manejo, o preparo do solo talvez seja a atividade que mais influi no seu comportamento físico, pois atua diretamente na estrutura do solo. Além das modificações na porosidade e na densidade, o manejo provoca alterações na estrutura do solo que afetam a retenção de água e a resistência mecânica, entre outros efeitos (Silva et al., 1994). 
Atualmente no Brasil, como no resto do mundo, há uma grande preocupação com o aumento das áreas agrícolas com problemas de compactação, o que em grande parte se deve às operações mecanizadas realizadas sem considerar a umidade do solo (Silva et al., 2000).

No Cerrado, em áreas irrigadas, essa preocupação é ainda maior (Freitas, 1992), tendo em vista que ao se procurar otimizar o uso dos solos e dos equipamentos de irrigação, na maioria das vezes não são observadas as condições adequadas de umidade para a operação das máquinas no campo. Além disso, há uma carência de informações quanto à capacidade de suporte de carga desses solos que possam ser usadas como subsídio na tomada de decisão sobre a realização ou não de uma determinada operação mecanizada.

A compressão dos solos agrícolas, decorrente das operações mecanizadas, pode alterar a distribuição e o tamanho dos poros e, conseqüentemente, a tensão com que a água é retida (Larson \& Gupta, 1980). Por sua vez, a variação da tensão da água no solo afeta seu comportamento compressivo (Horn \& Lebert, 1994). Apesar disso, são poucos os trabalhos que relacionam tal dependência, particularmente em relação aos Latossolos brasileiros (Larson \& Gupta, 1980; Carpenedo, 1994).

A pressão de preconsolidação tem sido usada para a predição da capacidade de suporte de carga do solo (Larson \& Gupta, 1980; Kondo \& Dias Junior, 1999; Silva et al., 2000; Imhoff et al., 2001), uma vez que esse parâmetro quantifica a maior pressão que o solo já sofreu no passado e, portanto, representa o limite máximo de carga suportado antes que a compactação adicional ocorra (Dias Junior \& Pierce, 1996). A pressão de preconsolidação pode ser determinada das curvas de compressão do solo, obtidas dos ensaios de compressão uniaxial (Dias Junior, 1994; Horn \& Lebert, 1994; Silva et al., 2000; Imhoff et al., 2001).

A estimativa é que as pressões médias aplicadas aos solos pelas máquinas agrícolas se situam entre 50 e 300 kPa (Lebert \& Horn, 1991; Carpenedo, 1994; Silva et al., 2000). Os reboques e caminhões podem exercer pressões entre 300 e $600 \mathrm{kPa}$ (Carpenedo, 1994; Silva et al., 2000).

O objetivo deste trabalho foi avaliar as alterações estruturais e o comportamento compressivo de um
Latossolo Vermelho distrófico sob Cerrado, plantio direto e preparo com arado de discos, após duas décadas de uso.

\section{Material e Métodos}

O experimento foi realizado em um Latossolo Vermelho distrófico típico (Embrapa, 1999), em área da Embrapa-Centro de Pesquisa Agropecuária dos Cerrados, Planaltina, DF (latitude $15^{\circ} 36^{\prime} \mathrm{S}$, longitude $47^{\circ} 42^{\prime} \mathrm{W}$ e altitude de $1.014 \mathrm{~m}$ ). Durante 20 anos a área foi manejada, em parcelas experimentais de 50x25 m, com arado de discos e plantio direto. Uma área sob vegetação natural de Cerrado foi selecionada como referência. Em 1979, antes da instalação, toda a área destinada ao manejo com culturas foi preparada com arado de discos, ocasião em que foram incorporados calcário e fertilizantes contendo $\mathrm{P}$ e potássio.

No segundo ano agrícola (1980/1981), a área foi dividida em parcelas de 50x $25 \mathrm{~m}$, recebendo cada uma os seguintes tratamentos de manejo: 1: plantio direto; 2 : aração com incorporação dos restos culturais após colheita da cultura principal (abril/maio) e 3: aração com incorporação dos restos culturais na época que antecede o plantio da cultura principal (setembro/outubro). Apenas os tratamentos $1 \mathrm{e}$ 3 foram selecionados para este trabalho.

$O$ uso e a rotação de culturas que vêm sendo implementados nessas parcelas, manejadas com arado de discos e plantio direto desde 1979, são apresentados na Tabela 1.

No mês de fevereiro de 1999, por ocasião do florescimento da soja, e em outubro do mesmo ano, após preparo do solo com arado, no tratamento com preparo convencional, foram feitas amostragens em três pontos de uma linha diagonal, distantes entre si $21 \mathrm{~m}$, com os pontos extremos a $6 \mathrm{~m}$ da borda limítrofe da parcela. Foram coletadas, nas profundidades de $0-5 \mathrm{~cm}$ e $20-30 \mathrm{~cm}, 15$ amostras indeformadas em cada época, por parcela, sendo três em anéis de $25 \mathrm{~mm}$ de diâmetro e $50 \mathrm{~mm}$ de altura para obtenção das curvas de retenção de água pelo método da centrífuga (Freitas Júnior \& Silva, 1984), nas tensões de água de $-1,-2,-4,-6,-8,-10,-33,-60,-80,-100,-500$ e $-1.500 \mathrm{kPa}$, e 12, em anéis com $63 \mathrm{~mm}$ de diâmetro e $25 \mathrm{~mm}$ de altura para análises físico-mecânicas.

A discriminação de poros por tamanho foi realizada segundo o modelo de Bouma (1973). O diâmetro dos poros foi calculado pela equação de ascensão capilar: $\psi \mathrm{m}=4 \sigma \operatorname{Cos} \theta / \phi g \mathrm{~d}$,

em que: $\psi$ m é a tensão da água no solo $(\mathrm{kPa})$; $\sigma$ é a tensão superficial da água $\left(0,727\right.$ bar $\mu \mathrm{m}$ a $\left.20^{\circ} \mathrm{C}\right) ; \theta$ é o ângulo de contato entre o menisco e a parede do tubo capilar (considerado como 0); $\phi$ é o diâmetro do poro $(\mu \mathrm{m})$ e dé a densidade da água $\left(\mathrm{Mg} \mathrm{m}^{-3}\right)$. 
Foram coletadas 24 subamostras deformadas para formarem 12 amostras compostas para caracterização dos óxidos pelo ataque sulfúrico; textura pelo método da pipeta (Tabela 2); densidade de partículas (Dp) pelo método do picnômetro; e carbono orgânico (Corg.) obtido da oxidação da matéria orgânica via úmida (Embrapa, 1997). A matéria orgânica foi calculada pela expressão $\mathrm{MO}=\mathrm{C}$ org. X 1,724. Também nas amostras indeformadas foram determinados o limite de plasticidade (LP) (Sowers, 1965) e o limite de contração (LC) (Bowles, 1986), cujos valores de umidade foram convertidos em tensão de água. Agregados com diâmetro entre 4,76 e 7,93 $\mathrm{mm}$ foram obtidos por peneiramento de material indeformado, amostrado nas profundidades de $0-5 \mathrm{~cm}$ e $20-30 \mathrm{~cm}$ do solo, conforme Kemper \& Rosenau (1986). A estabilidade de agregados foi determinada por peneiramento em água, após pré-umedecimento lento, por capilaridade. O diâmetro

Tabela 1. Histórico de uso e rotação de culturas na área experimental da Embrapa-Centro de Pesquisa Agropecuária dos Cerrados sob Latossolo Vermelho distrófico.

\begin{tabular}{llll}
\hline Ano & Cultura & \multicolumn{2}{c}{ Preparo do solo } \\
\cline { 3 - 4 } & & \multicolumn{1}{c}{ Parcela 1 } & \multicolumn{1}{c}{ Parcela 3 } \\
\hline $1979 / 80$ & Arroz & Convencional & Convencional \\
$1980 / 81$ & Arroz & Plantio direto & ADPC $^{(2)}$ \\
$1981 / 82$ & Soja & Plantio direto & ADPC \\
$1982 / 83$ & Guandu & Plantio direto & ADPC \\
$1983 / 86$ & & Pousio & Pousio \\
$1986 / 87$ & Soja & Plantio direto & ADPC \\
$1987 / 88$ & Soja & Plantio direto & ADPC \\
$1988 / 89$ & Soja e Milho & Plantio direto & Plantio direto \\
$1989 / 90$ & & Plausio & Pousio \\
$1990 / 91$ & Milho & Plantio direto & Plantio direto \\
$1991 / 92$ & Soja & Plantio direto & Plantio direto \\
$1992 / 93$ & Soja e Milho & Plantio direto & ADPC \\
$1993 / 94$ & Soja e Milho & Plantio direto & ADPC \\
$1994 / 95$ & Arroz & Plantio direto & ADPC \\
$1995 / 96$ & Soja & Plantio direto & ADPC \\
$1996 / 97$ & Milho & Plantio direto & ADPC \\
$1997 / 98$ & Soja & Plantio direto & ADPC \\
$1998 / 99$ & Soja & Plantio direto & ADPC \\
\hline
\end{tabular}

(1)Uso do arado de discos com duas passagens de grade leve, para incorporação de calcário e fertilizantes ( $\mathrm{P}$ e $\mathrm{K})$, apenas no início do experimento.

(2)Uso do arado de discos com incorporação dos restos culturais após colheita da cultura principal. ${ }^{(3)}$ Nesses anos as parcelas foram subdivididas em duas, metade da área cultivada com soja e metade com milho. médio geométrico (DMG) foi calculado segundo Kemper \& Rosenau (1986).

As amostras indeformadas, coletadas em anéis com $63 \mathrm{~mm}$ de diâmetro e $25 \mathrm{~mm}$ de altura, após serem equilibradas com água na tensão de -6 kPa pela unidade de sucção (Grohmann, 1960) e aparelho de Richards (Klute, 1986) nas tensões de $-33,-100$ e $-1.500 \mathrm{kPa}$, foram submetidas ao ensaio de compressão uniaxial (Dias Junior, 1994), usando um consolidômetro da marca Boart Longyear, cuja aplicação das pressões se fez por ar comprimido. As tensões extremas de -6 e $-1.500 \mathrm{kPa}$ foram escolhidas por serem consideradas como de equilíbrio da água na capacidade de campo e ponto de murcha permanente, respectivamente (Santos, 1997). Foram aplicadas em cada amostra 25, 50, $100,200,400,800$ e $1.600 \mathrm{kPa}$ de pressão, com a aplicação de cada uma delas feita até que $90 \%$ da deformação máxima fosse alcançada (Holtz \& Kovacs, 1981), aplicando-se logo a seguir pressão mais elevada.

As amostras previamente submetidas à tensão de - $6 \mathrm{kPa}$ foram utilizadas na determinação da microporosidade (Grohmann, 1960; Oliveira, 1968). $\mathrm{O}$ volume total de poros (VTP) foi calculado pela expressão: VTP = (1 - Ds/Dp) 100, sendo Ds: densidade do solo e Dp: densidade de partículas; a macroporosidade foi determinada pela diferença entre VTP (calculada) e microporosidade.

As curvas de compressão do solo foram obtidas plotando-se a pressão aplicada no eixo das abscissas (escala logarítmica) e a densidade do solo correspondente a cada pressão aplicada no eixo das ordenadas (escala decimal). A partir das curvas de compressão, foram obtidas as pressões de preconsolidação (Dias Junior \& Pierce, 1995).

As pressões de preconsolidação $\left(\sigma_{\mathrm{p}}\right)$ foram plotadas, em escala decimal, no eixo das ordenadas, e as tensões de água retida $(\psi \mathrm{m})$, em escala logarítmica, no eixo das abscissas, obtendo-se os modelos de compressibilidade.

As comparações das equações entre os sistemas, relacionando pressão de preconsolidação e tensão de água no solo, foram feitas de acordo com Snedecor \& Cochran (1989).

Os resultados das análises físicas, mecânicas e de matéria orgânica foram submetidos à análise de variância, segundo delineamento experimental inteiramente casualizado, e à comparação das médias dos sistemas de manejo pelo teste de Scott-Knott a 5\% de probabilidade.

Tabela 2. Atributos químicos e físicos nas profundidades de $0-5 \mathrm{~cm}$ e $20-30 \mathrm{~cm}$ do Latossolo Vermelho distrófico ${ }^{(1)}$.

\begin{tabular}{|c|c|c|c|c|c|c|c|c|c|}
\hline \multirow{2}{*}{$\begin{array}{l}\text { Prof. } \\
\text { (cm) }\end{array}$} & \multicolumn{3}{|c|}{ Ataque sulfúrico } & \multicolumn{4}{|c|}{ Granulometria } & \multirow[t]{2}{*}{$\mathrm{Ki}$} & \multirow[t]{2}{*}{$\mathrm{Kr}$} \\
\hline & $\mathrm{SiO}_{2}$ & $\mathrm{Al}_{2} \mathrm{O}_{3}$ & $\mathrm{Fe}_{2} \mathrm{O}_{3}$ & $\mathrm{~A}$ & $S$ & $\mathrm{AF}$ & $\mathrm{AG}$ & & \\
\hline $0-5$ & 170 & 230 & 86 & 530 & 131 & 210 & 129 & 1,3 & 1,0 \\
\hline $20-30$ & 188 & 248 & 85 & 530 & 120 & 220 & 130 & 1,3 & 1,1 \\
\hline
\end{tabular}

(1) A: argila; S: silte; AF: areia fina; $\mathrm{AG}$ : areia grossa; Ki: relação molecular $\mathrm{SiO}_{2}: \mathrm{Al}_{2} \mathrm{O}_{3} ; \mathrm{Kr}$ : relação molecular $\mathrm{SiO}_{2}:\left(\mathrm{Al}_{2} \mathrm{O}_{3}+\mathrm{Fe}_{2} \mathrm{O}_{3}\right)$. 


\section{Resultados e Discussão}

Independentemente da profundidade e da época das coletas das amostras, o solo sob Cerrado apresentou menor densidade e maior relação macroporos:microporos comparado ao plantio direto e preparo com arado de discos, resultado da interferência do homem por meio da ação de máquinas e equipa- mentos, que alteraram essas propriedades do solo nestes dois sistemas de plantio (Tabela 3).

Na profundidade de $0-5 \mathrm{~cm}$, nas duas datas de coleta das amostras no plantio direto, após duas décadas de manejo contínuo, a relação macroporos:microporos foi igual à do preparo com arado de discos, que movimenta o solo e o deixa mais solto, pelo menos temporariamente. Isto ocorreu, prova-

Tabela 3. Distribuição temporal de atributos físicos e de matéria orgânica do Latossolo Vermelho distrófico, sob diferentes sistemas de manejo, em duas épocas de coleta e em duas profundidades ${ }^{(1)}$.

\begin{tabular}{|c|c|c|c|c|c|c|}
\hline Sistema & $\begin{array}{c}\text { Ds } \\
\left(\mathrm{kg} \mathrm{dm}^{-3}\right)\end{array}$ & Mac:Mic & LP & LC & $\begin{array}{c}\mathrm{MO} \\
\left(\mathrm{g} \mathrm{kg}^{-1}\right)\end{array}$ & $\begin{array}{l}\mathrm{DMG} \\
(\mathrm{mm})\end{array}$ \\
\hline & \multicolumn{6}{|c|}{ Profundidade de 0-5 cm - Fevereiro de 1999} \\
\hline Cerrado & $\begin{array}{c}0,90 \mathrm{~B} \\
(6,7)\end{array}$ & $\begin{array}{l}1,08 \mathrm{~A} \\
(16,3)\end{array}$ & $\begin{array}{c}0,30 \mathrm{~A} \\
(6) \\
{[-11 \mathrm{kPa}]}\end{array}$ & $\begin{array}{c}0,20 \mathrm{~A} \\
(10) \\
{[-2.010 \mathrm{kPa}]}\end{array}$ & $\begin{array}{l}50 \mathrm{~A} \\
(33)\end{array}$ & \\
\hline Plantio direto & $\begin{array}{c}1,12 \mathrm{~A} \\
(7,4)\end{array}$ & $\begin{array}{l}0,59 \mathrm{~B} \\
(21,7)\end{array}$ & $\begin{array}{c}0,27 \mathrm{~A} \\
(6) \\
{[-16 \mathrm{kPa}]}\end{array}$ & $\begin{array}{c}0,21 \mathrm{~A} \\
(10) \\
{[-188 \mathrm{kPa}]}\end{array}$ & $\begin{array}{c}51 \mathrm{~A} \\
(3)\end{array}$ & \\
\hline \multirow[t]{2}{*}{ discos } & $\begin{array}{c}1,15 \mathrm{~A} \\
(5,1)\end{array}$ & $\begin{array}{c}0,66 \mathrm{~B} \\
(8,4)\end{array}$ & $\begin{array}{c}0,27 \mathrm{~A} \\
(6) \\
{[-17 \mathrm{kPa}]}\end{array}$ & $\begin{array}{c}0,22 \mathrm{~A} \\
(7) \\
{[-102 \mathrm{kPa}]}\end{array}$ & $\begin{array}{l}24 \mathrm{~B} \\
(13)\end{array}$ & \\
\hline & \multicolumn{6}{|c|}{ Profundidade de 0-5 cm - Outubro de 1999} \\
\hline Cerrado & $\begin{array}{c}0,91 \mathrm{~B} \\
(5,0)\end{array}$ & $\begin{array}{l}1,30 \mathrm{~A} \\
(16,3)\end{array}$ & & & $\begin{array}{l}48 \mathrm{~A} \\
(32)\end{array}$ & $\begin{array}{c}4,4 \mathrm{~A} \\
(12)\end{array}$ \\
\hline Plantio direto & $\begin{array}{c}1,09 \mathrm{~A} \\
(9,0)\end{array}$ & $\begin{array}{l}0,61 \mathrm{~B} \\
(36,8)\end{array}$ & & & $\begin{array}{l}48 \mathrm{~A} \\
(11)\end{array}$ & $\begin{array}{c}3,6 \mathrm{~B} \\
(8)\end{array}$ \\
\hline $\begin{array}{l}\text { Arado de } \\
\text { discos }\end{array}$ & $\begin{array}{c}1,13 \mathrm{~A} \\
(5,0)\end{array}$ & $\begin{array}{l}0,72 B \\
(11,2) \\
\end{array}$ & & & $\begin{array}{l}28 \mathrm{~B} \\
(11) \\
\end{array}$ & $\begin{array}{c}3,3 \mathrm{C} \\
(6) \\
\end{array}$ \\
\hline Cerrado & $\begin{array}{c}1,03 \mathrm{C} \\
(4,2)\end{array}$ & $\begin{array}{c}\text { Profi } \\
0,83 \mathrm{~A} \\
(6,9)\end{array}$ & $\begin{array}{c}\text { de } 20-30 \mathrm{c} \\
0,30 \mathrm{~A} \\
(6) \\
{[-6 \mathrm{kPa}]}\end{array}$ & $\begin{array}{c}\text { reiro de } 1999 \\
0,20 \mathrm{~A} \\
(14) \\
{[-1.400 \mathrm{kPa}]}\end{array}$ & $\begin{array}{c}24 \mathrm{~A} \\
(9)\end{array}$ & \\
\hline Plantio direto & $\begin{array}{c}1,15 \mathrm{~B} \\
(5,1)\end{array}$ & $\begin{array}{c}0,61 \mathrm{~B} \\
(0,6)\end{array}$ & $\begin{array}{c}0,29 \mathrm{~A} \\
(6) \\
{[-10 \mathrm{kPa}]}\end{array}$ & $\begin{array}{c}0,21 \mathrm{~A} \\
(10) \\
{[-188 \mathrm{kPa}]}\end{array}$ & $\begin{array}{c}27 \mathrm{~A} \\
(6)\end{array}$ & \\
\hline \multirow[t]{2}{*}{$\begin{array}{l}\text { Arado de } \\
\text { discos }\end{array}$} & $\begin{array}{c}1,23 \mathrm{~A} \\
(4,6)\end{array}$ & $\begin{array}{l}0,50 \mathrm{~B} \\
(10,9)\end{array}$ & $\begin{array}{c}0,29 \mathrm{~A} \\
(4) \\
{[-8 \mathrm{kPa}]} \\
\end{array}$ & $\begin{array}{c}0,21 \mathrm{~A} \\
(6) \\
{[-175 \mathrm{kPa}]} \\
\end{array}$ & $\begin{array}{l}27 \mathrm{~A} \\
(12)\end{array}$ & \\
\hline & \multicolumn{6}{|c|}{ Profundidade de 20-30 cm - Outubro de 1999} \\
\hline Cerrado & $\begin{array}{c}1,02 \mathrm{C} \\
(5,2)\end{array}$ & $\begin{array}{c}1,50 \mathrm{~A} \\
(5,3)\end{array}$ & & & $\begin{array}{c}29 \mathrm{~A} \\
(2)\end{array}$ & $\begin{array}{c}4,3 \mathrm{~A} \\
(15)\end{array}$ \\
\hline Plantio direto & $\begin{array}{c}1,14 \mathrm{~B} \\
(5,8)\end{array}$ & $\begin{array}{l}0,59 \mathrm{~B} \\
(16,1)\end{array}$ & & & $\begin{array}{c}29 \mathrm{~A} \\
(5)\end{array}$ & $\begin{array}{c}4,3 \mathrm{~A} \\
(10)\end{array}$ \\
\hline $\begin{array}{l}\text { Arado de } \\
\text { discos }\end{array}$ & $\begin{array}{c}1,22 \mathrm{~A} \\
(7,7)\end{array}$ & $\begin{array}{l}0,56 \mathrm{~B} \\
(31,9) \\
\end{array}$ & & & $\begin{array}{l}28 \mathrm{~A} \\
(10)\end{array}$ & $\begin{array}{c}4,2 \mathrm{~A} \\
(8)\end{array}$ \\
\hline
\end{tabular}

(1)Para cada parâmetro analisado, dentro de cada profundidade e época, médias seguidas pelas mesmas letras, nas colunas, não diferem entre si pelo teste de Scott-Knott a 5\% de probabilidade; Ds: densidade do solo (média de 12 repetições); Mac:Mic: relação entre macroporos e microporos (média de seis repetições); LP: limite de plasticidade (média de três repetições); LC: limite de contração (média de três repetições); MO: matéria orgânica (média de seis repetições); DMG: diâmetro médio geométrico (média de três repetições); número entre parênteses: coeficientes de variação (\%); número entre colchetes: tensão de água no solo em que se encontram os limites de plasticidade e contração. 
velmente, por causa do maior teor de matéria orgânica no sistema plantio direto, estatisticamente idêntico ao do solo sob Cerrado (Tabela 3), corroborando as observações de Fernandes et al. (1983) de que a diminuição da densidade e a melhoria da estrutura na camada superficial de solos sob plantio direto, com o passar dos anos, são causadas, em parte, pelo aumento do conteúdo de matéria orgânica.

Apesar de a densidade do solo, macroporosidade e a relação macroporos:microporos serem propriedades muito utilizadas como indicadoras do estado de estruturação do solo (Gupta \& Allmaras, 1987; Imhoff et al., 2001), pelo fato de a densidade do solo ser influenciada pelos seus constituintes, entre eles a textura e a matéria orgânica, esse parâmetro pode mostrar uma correlação limitada com o crescimento das plantas (Campbell, 1994). Quanto ao uso da macroporosidade e da relação macroporos: microporos, não existe consenso na classificação, variando nos diferentes estudos os valores limites de diâmetro entre os parâmetros macroporosidade e microporosidade (Bouma, 1991; Prevedello, 1996), embora exista tendência de se adotar o diâmetro de $50 \mu \mathrm{m}$ (Oliveira, 1968; Tormena et al., 1998). Desta forma, em estudos comparativos entre sistemas de manejo, provavelmente a discriminação da distribuição de poros no solo por tamanho seja o mais indicado.

Os dados da Tabela 4 revelam a dificuldade de diagnóstico de alterações na estrutura de um solo submetido a diferentes sistemas de uso ou manejo, quando normalmente se considera apenas o volume de poros maiores (macroporos) e menores (microporos) que $50 \mu \mathrm{m}$ (Oliveira, 1968; Tormena et al., 1998).

As maiores diferenças na estrutura foram encontradas em poros maiores que $145 \mu \mathrm{m}$, correspondente a uma parte da porosidade de aeração, e em poros entre 50 e 9,0 $\mu \mathrm{m}$, uma fração da porosidade de retenção de água, quando se considera a classificação de Oliveira (1968), cujo limite entre macroporos e microporos é de $50 \mu \mathrm{m}$ de diâmetro (Tabela 3). No solo sob Cerrado, observou-se maior volume de poros maiores que $145 \mu \mathrm{m}$ (aeração) e menor volume de poros responsáveis pelo armazenamento de água disponível para as plantas.

Na profundidade de $0-5 \mathrm{~cm}$, o plantio direto, em relação ao sistema de preparo com arado de discos, foi o que mais afetou o arranjo das classes de poros do solo, causando maior redução no volume de poros com diâmetros superiores a $145 \mu \mathrm{m}$, e o desenvolvimento de maior volume de poros entre $50 \mathrm{e}$ 9,0 $\mu \mathrm{m}$ de diâmetro. Tal resultado é benéfico (valores médios de poros), pela maior retenção de água pelo solo, sem danos na sua porosidade (Tabela 3), quando se considera que um solo em condições ideais deve apresentar $1 / 3$ da porosidade total formada por macroporos e os 2/3 restantes por microporos, estabelecendo uma relação macroporos:microporos igual a 0,5 (Kiehl, 1979). Já na profundidade de $20-30$ cm, o preparo com arado de discos foi o que mais afetou a estrutura do solo, fato constatado pela maior densidade do solo, causada pela ação do implemento na

Tabela 4. Distribuição de poros $\left(\mathrm{m}^{3} \mathrm{~m}^{-3}\right)$ por tamanho em duas profundidades de um Latossolo Vermelho distrófico, sob diferentes sistemas de manejo ${ }^{(1)}$

\begin{tabular}{|c|c|c|c|c|c|c|c|}
\hline \multirow[t]{2}{*}{ Sistema } & \multicolumn{6}{|c|}{ Diâmetro dos poros $(\mu \mathrm{m})$} & \multirow{2}{*}{$\begin{array}{l}\text { Volume total } \\
\text { de poros }\end{array}$} \\
\hline & $>145$ & $145-73$ & $73-50$ & $50-9,0$ & $9,0-2,9$ & $<2,9$ & \\
\hline & \multicolumn{7}{|c|}{ Profundidade de $0-5 \mathrm{~cm}$} \\
\hline Cerrado & $0,25 \mathrm{~A}$ & $0,07 \mathrm{~A}$ & $0,02 \mathrm{~B}$ & $0,05 \mathrm{~B}$ & $0,02 \mathrm{~B}$ & $0,23 \mathrm{~B}$ & 0,64 \\
\hline Plantio direto & $0,08 \mathrm{C}$ & $0,03 \mathrm{~A}$ & $0,03 \mathrm{~A}$ & $0,16 \mathrm{~A}$ & $0,03 \mathrm{~A}$ & $0,25 \mathrm{~A}$ & 0,58 \\
\hline \multirow[t]{2}{*}{ Arado de discos } & $0,13 \mathrm{~B}$ & $0,05 \mathrm{~A}$ & $0,03 \mathrm{~A}$ & $0,08 \mathrm{~B}$ & $0,03 \mathrm{~A}$ & $0,24 \mathrm{~A}$ & 0,56 \\
\hline & \multicolumn{7}{|c|}{ Profundidade de $20-30 \mathrm{~cm}$} \\
\hline Cerrado & $0,26 \mathrm{~A}$ & $0,07 \mathrm{~A}$ & $0,02 \mathrm{~A}$ & $0,05 \mathrm{~B}$ & $0,02 \mathrm{~B}$ & $0,23 \mathrm{~A}$ & 0,65 \\
\hline Plantio direto & $0,12 \mathrm{~B}$ & $0,06 \mathrm{~A}$ & $0,04 \mathrm{~A}$ & $0,08 \mathrm{~A}$ & $0,03 \mathrm{~A}$ & $0,25 \mathrm{~A}$ & 0,58 \\
\hline Arado de discos & $0,09 \mathrm{~B}$ & $0,03 \mathrm{~A}$ & $0,03 \mathrm{~A}$ & $0,08 \mathrm{~A}$ & $0,03 \mathrm{~A}$ & $0,27 \mathrm{~A}$ & 0,53 \\
\hline
\end{tabular}

${ }^{(1)}$ Dentro de cada profundidade, médias seguidas pela mesma letra, nas colunas, não diferem entre si pelo teste de Scott-Knott a 5\% de probabilidade. 
profundidade especificada, a exemplo do relatado por Tormena et al. (1998), mantendo, entretanto, a relação macroporos:microporos acima de 0,5 (Tabela 3). Salienta-se que a maioria das plantas desenvolve satisfatoriamente seu sistema radicular quando a porcentagem de macroporos (poros maiores que $50 \mu \mathrm{m}$ ) está acima de $0,10 \mathrm{~m}^{3} \mathrm{~m}^{-3}$ (Kiehl, 1979; Gupta \& Allmaras, 1987), condição verificada no solo sob todos os sistemas de uso ou manejo, independentemente da profundidade e época de amostragem (Tabela 4).

Na profundidade de $0-5 \mathrm{~cm}$, na seqüência Cerrado e plantio direto, foram observados valores de $\mathrm{DMG}$ maiores do que o do preparo com arado de discos (Tabela 3), concordando com Beutler et al. (2001). Isso pode ser decorrente dos mais altos teores de $\mathrm{C}$ orgânico presentes nesses sistemas (Tabela 3), uma vez que o $\mathrm{C}$ orgânico tem papel preponderante na formação e preservação de macroagregados no solo, por ser eficiente agente cimentante (Campos et al., 1995). Apesar da reconhecida participação do C orgânico na agregação do solo, os maiores valores de DMG na camada superficial do solo nos sistemas plantio direto e Cerrado foram resultantes, também, da nula ou pequena movimentação do solo em relação ao preparo com arado de discos. Não foram notadas diferenças estatísticas nos valores de DMG entre sistemas, na profundidade de $20-30 \mathrm{~cm}$ em razão dos menores teores de C orgânico (Tabela 3).

As equações relacionando a pressão de preconsolidação $\left(\sigma_{\mathrm{p}}\right)$ com a tensão de água no solo $(\psi \mathrm{m})$ foram tratadas conjuntamente, pelo fato de não terem sido observadas diferenças estatísticas entre as regressões que modelam o comportamento compressivo do solo em cada sistema de manejo e em cada profundidade, nas duas épocas estudadas (Figura 1). Em relação às tensões de água estudadas, independentemente da profundidade, as menores e maiores pressões de preconsolidação (estimativa da capacidade suporte de carga do solo) foram encontradas na tensão de água no solo de $-6 \mathrm{kPa}$ e -1.500 kPa, respectivamente, em concordância com Soane (1990) e Carpenedo (1994), que destacam a dependência existente entre essas propriedades ( $\mathrm{Fi}$ gura 1). Na profundidade de $0-5 \mathrm{~cm}$, pela comparação das equações $\sigma_{\mathrm{p}}=\mathrm{f}(\psi \mathrm{m})$, os solos sob plantio direto e sob Cerrado superaram o solo sob preparo com arado de discos em suporte de carga $(\mathrm{F}=3$; $\mathrm{p}<0,05$ e $\mathrm{F}=2 ; \mathrm{p}<0,05$, respectivamente) (Figura 1 ). Na profundidade de $20-30 \mathrm{~cm}$, o solo sob preparo com arado de discos apresentou maior suporte de carga em relação aos tratamentos plantio direto e Cerrado $(F=2 ; p<0,05$ e $F=2 ; p<0,05$, respectivamente). Não foram encontradas diferenças estatísticas no comportamento compressivo do solo sob plantio direto em relação ao solo sob Cerrado, nas profundidades estudadas.

O método estatístico utilizado (Snedecor \& Cochran, 1989) é limitado quanto a comparações entre regressões, não sendo indicado para análises pontuais, o que dificulta a comparação do comportamento compressivo do solo sob sistemas de manejo, em diferentes tensões de água no solo. Assim, quando os dados de comportamento compressivo em cada tensão de água foram submetidos à análise de

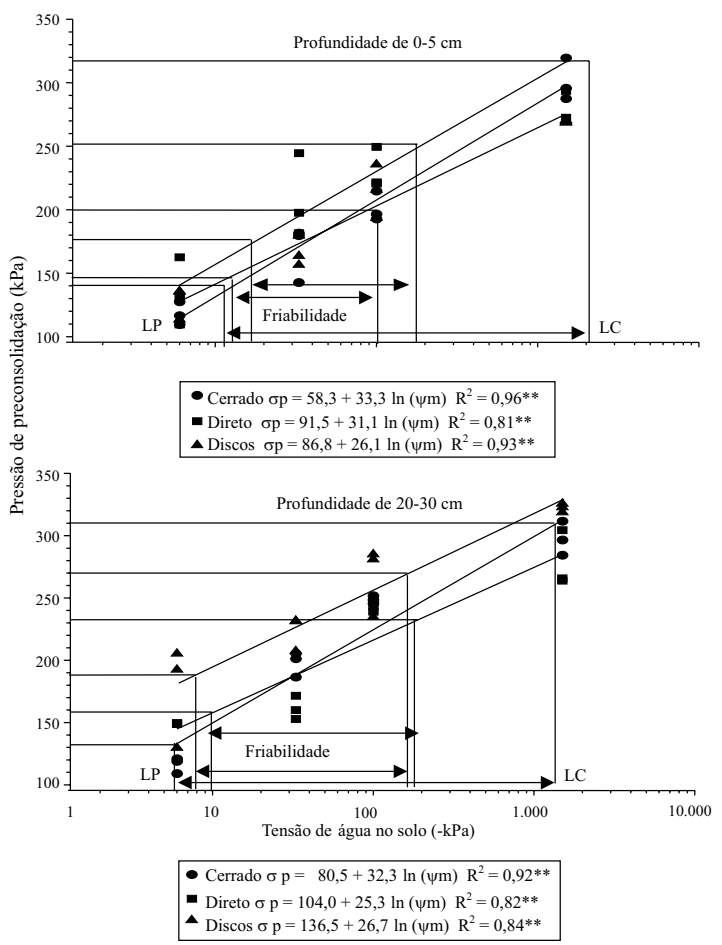

Figura 1. Comportamento compressivo do solo [pressão de preconsolidação $\left(\sigma_{\mathrm{p}}\right)$ em razão da tensão de água no solo $(\psi \mathrm{m})]$ e zona de friabilidade em amostras coletadas em fevereiro e outubro de 1999, nas profundidades de 0-5 cm e 20-30 cm do Latossolo Vermelho argiloso, sob Cerrado (•), plantio direto $(\mathbf{\square})$ e preparo com arado de discos (ム). LP: limite de plasticidade; LC: limite de contração. **Significativo a $1 \%$ de probabilidade. 
variância, constatou-se que, nas duas profundidades, as diferenças de comportamento compressivo entre os sistemas só ocorreram na tensão de água de $-1.500 \mathrm{kPa}(\mathrm{F}=3 ; \mathrm{p}<0,05$, para a profundidade de $0-5 \mathrm{~cm} ;$ e $\mathrm{F}=8 ; \mathrm{p}<0,01$, para a profundidade de 20-30 cm).

Na profundidade de $0-5 \mathrm{~cm}$, na tensão de água de -1.500 kPa, o solo sob Cerrado e sob plantio direto suportou mais carga em relação à condição de preparo com arado de discos (Tabela 5). Isto ocorreu, provavelmente, em virtude da maior estabilidade de agregados do solo (Tabela 3), acrescida da maior compacidade do solo no sistema plantio direto, pelo menor volume de poros com diâmetro maior que $145 \mu \mathrm{m}$ (Tabela 4). Acredita-se que a grande adição de resíduos e a ausência de revolvimento nesses sistemas refletem em maior coesão entre as partículas e agregados do solo (Soane, 1990), principalmente em tensões mais baixas de umidade (Carpenedo, 1994).

Na profundidade de $20-30 \mathrm{~cm}$, o solo sob preparo com arado de discos suportou mais carga em relação aos demais sistemas, na tensão de água de $-1.500 \mathrm{kPa}$ (Tabela 5). Tendo em vista que o solo nessa profundidade, sob todos os sistemas de uso e manejo, apresentou teores estatisticamente iguais de matéria orgânica, e valor estatisticamente superior quanto à densidade, quando sob preparo com arado de discos (Tabela 3), as diferenças de comportamento compressivo devem ser resultantes do aumento da compacidade do solo, provocado pela ação do implemento na profundidade especificada. Segundo

Tabela 5. Pressão de preconsolidação $\left(\sigma_{\mathrm{p}}\right)$ nos potenciais de água estudados e nas duas profundidades do Latossolo Vermelho distrófico ${ }^{(1)}$

\begin{tabular}{lcccc}
\hline \multirow{2}{*}{ Sistema } & \multicolumn{4}{c}{ Potenciais de água no solo (kPa) } \\
\cline { 2 - 5 } & -6 & -33 & -100 & -1.500 \\
\hline \multirow{4}{c}{ Profundidade de 0-5 cm } \\
Cerrado & $125,3 \mathrm{~A}$ & $182,2 \mathrm{~A}$ & $212,3 \mathrm{~A}$ & $314,1 \mathrm{~A}$ \\
Plantio direto & $143,1 \mathrm{~A}$ & $213,3 \mathrm{~A}$ & $238,4 \mathrm{~A}$ & $320,1 \mathrm{~A}$ \\
Arado de discos & $133,9 \mathrm{~A}$ & $177,6 \mathrm{~A}$ & $212,3 \mathrm{~A}$ & $275,4 \mathrm{~B}$ \\
\hline \multicolumn{4}{c}{ Profundidade de $20-30 \mathrm{~cm}$} \\
Cerrado & $120,7 \mathrm{~A}$ & $202,2 \mathrm{~A}$ & $253,0 \mathrm{~A}$ & $302,0 \mathrm{~B}$ \\
Plantio direto & $153,6 \mathrm{~A}$ & $165,9 \mathrm{~A}$ & $248,4 \mathrm{~A}$ & $282,5 \mathrm{~B}$ \\
Arado de discos & $180,2 \mathrm{~A}$ & $227,9 \mathrm{~A}$ & $271,3 \mathrm{~A}$ & $326,8 \mathrm{~A}$ \\
\hline (1)Dentro de cada profundidade, médias seguidas pela mesma letra, nas co- \\
lunas, não diferem entre si pelo teste de Scott-Knott a 5\% de probabilidade; \\
os dados representam médias de duas épocas de coleta.
\end{tabular}

Imhoff et al. (2001), um incremento na densidade do solo leva ao aumento das forças de fricção ou coesão, e dos pontos de contato entre as partículas do solo, o que, conforme Guérif (1994), condiciona o aumento da capacidade de suporte de carga. Soane (1990) e Carpenedo (1994) constataram que o tipo e o grau de estrutura do solo têm forte influência na capacidade de suporte de carga.

Quanto à recomendação para o trabalho com máquinas na zona de friabilidade (Hillel, 1982), e considerando a pressão de preconsolidação como a maior pressão que deve ser aplicada ao solo para evitar que a compactação adicional ocorra (Kondo \& Dias Junior, 1999), constata-se que, na profundidade de 0-5 cm, os valores de pressão a serem aplicados ao solo para evitar a compactação variaram de 144 a $321 \mathrm{kPa}$, para tensões de água entre - 11 e - $2.010 \mathrm{kPa}$, em solo sob Cerrado; 180 a $258 \mathrm{kPa}$, para tensões de água no solo entre -16 e - $188 \mathrm{kPa}$, sob plantio direto; e 152 a $209 \mathrm{kPa}$, para tensões de água entre -12 e -102 kPa, sob preparo com arado de discos (Figura 1).

Na profundidade de $20-30 \mathrm{~cm}$, os valores de pressão que devem ser aplicados ao solo para evitar a compactação variaram de 138 a $314 \mathrm{kPa}$, para tensões de água entre - 6 e $-1.397 \mathrm{kPa}$, em solo sob Cerrado; 162 a $237 \mathrm{kPa}$, para tensões de água entre -10 e -188 kPa, sob plantio direto; e 193 a $274 \mathrm{kPa}$, para tensões de água entre -8 e $-175 \mathrm{kPa}$, sob preparo com arado de discos (Figura 1).

Considerando 50 e $300 \mathrm{kPa}$ como os valores de pressão média aplicada aos solos pelas máquinas agrícolas (Lebert \& Horn, 1991; Carpenedo, 1994; Silva et al., 2000), verifica-se que há necessidade de cautela na tomada de decisão do momento mais adequado para o uso de máquina no campo, pois mesmo estando o solo sob tensões de água dentro da zona de friabilidade (Figura 1), pode ocorrer compactação do solo nessa zona, pois a capacidade de suporte de carga do solo pode ser excedida (Kondo \& Dias Junior, 1999).

\section{Conclusões}

1. As tensões de água no solo afetam a pressão de preconsolidação nos três sistemas estudados, sendo os valores mais elevados nas tensões mais baixas. 
2. Na profundidade de $0-5 \mathrm{~cm}$ e sob a tensão de -1.500 kPa, os solos sob Cerrado e sob plantio direto apresentam maior capacidade de suporte de carga quando comparados ao solo preparado com arado de discos.

3. Na profundidade de $20-30 \mathrm{~cm}$ e sob a tensão de $-1.500 \mathrm{kPa}$, o solo sob preparo com arado de discos apresenta maior capacidade de suporte de carga quando comparado aos solos sob plantio direto e sob Cerrado.

\section{Referências}

BEUTLER, A. N.; SILVA, M. L. N.; CURI, N.; FERREIRA, M. M.; PEREIRA FILHO, I. A.; CRUZ, J. C. Agregação de Latossolo Vermelho distrófico típico relacionada com o manejo na região dos Cerrados no Estado de Minas Gerais. Revista Brasileira de Ciências do Solo, Viçosa, MG, v. 25, p. 129-136, 2001.

BOUMA, J. Guide to the study of water movement in soil pedons above the watertable. Madison: University of Wisconsin, 1973. $194 \mathrm{p}$.

BOUMA, J. Influence of soil macroporosity on environmental quality. Advances in Agronomy, San Diego, v. 46, p. 1-37, 1991 .

BOWLES, J. A. Engineering properties of soils and their measurements. 3rd ed. New York: McGraw-Hill, 1986. $218 \mathrm{p}$

CAMPBELL, D. J. Determination and use of soil bulk density in relation to soil compaction. In: SOANE, B. D.; OUWERKERK, C. van (Ed.). Soil compaction in crop production. Amsterdam: Elsevier, 1994. p. 113-139.

CAMPOS, B. C.; REINERT, D. J.; NICOLODI, R.; RUEDELL, J.; PETRERE, C. Estabilidade estrutural de um Latossolo Vermelho-Escuro distrófico após sete anos de rotação de culturas e sistemas de manejo do solo. Revista Brasileira de Ciência do Solo, Campinas, v. 19, p. 121-126, 1995.

CARPENEDO, V. Compressibilidade de solos em sistemas de manejo. 1994. 106 f. Tese (Doutorado em Ciência do Solo) - Universidade Federal do Rio Grande do Sul, Porto Alegre, 1994.

DIAS JUNIOR, M. S. Compression of three soils under long-term tillage and wheel traffic. 1994. 114 leaves. Thesis (Ph.D. in Crop and Soil Science) - Michigan State University, East Lansing, 1994.
DIAS JUNIOR, M. S.; PIERCE, F. J. A simple procedure for estimating preconsolidation pressure from soil compression curves. Soil Technology, Amsterdam, v. 8, p. 139-151, 1995.

DIAS JUNIOR, M. S.; PIERCE, F. J. O processo de compactação do solo e sua modelagem. Revista Brasileira de Ciência do Solo, Campinas, v. 20, p. 175-182, 1996.

EMBRAPA. Centro Nacional de Pesquisa de Solos (Rio de Janeiro, RJ). Manual de métodos de análises de solo. 2. ed. Rio de Janeiro, 1997. 212 p.

EMBRAPA. Centro Nacional de Pesquisa de Solos (Rio de Janeiro, RJ). Sistema brasileiro de classificação de solos. Brasília: Embrapa-CNPS/Embrapa-SPI, 1999. $412 \mathrm{p}$.

FERNANDES, B.; GALLOWAY, H. M.; BRONSON, R. D.; MANNERING, J. V. Efeito de três sistemas de preparo do solo na densidade aparente, na porosidade total e na distribuição dos poros, em dois solos (Typic Argiaquoll e Typic Hapludalf). Revista Brasileira de Ciência do Solo, Campinas, v. 7, p. 329-333, 1983.

FREITAS, P. L. Manejo físico do solo. In: COSTA, C. V.; BORGES, L. C. V. (Coord.). SIMPÓSIO SOBRE MANEJO E CONSERVAÇÃO DO SOLO NO CERRADO, 1990, Goiânia. Anais... Campinas: Fundação Cargill, 1992. p. 117-139.

FREITAS JÚNIOR, E.; SILVA, E. M. Uso da centrífuga para a determinação da curva de retenção de água no solo, em uma única operação. Pesquisa Agropecuária Brasileira, v. 19, n. 11, p. 1423-1428, nov. 1984.

GROHMANN, F. Distribuição e tamanho de poros em três tipos de solos do Estado de São Paulo. Bragantia, Campinas, v. 19, n. 21, p. 319-328, abr. 1960.

GUÉRIF, J. Effects of compaction on soil strength parameters. In: SOANE, B. D.; OUWERKERK, C. van. (Ed.). Soil compaction in crop production. Amsterdam: Elsevier, 1994. p. 191-214.

GUPTA, S. C.; ALLMARAS, R. R. Models to access the susceptibility of soil to excessive compaction. Advances in Soil Sciences, New York, v. 6, p. 65-100, 1987.

HILLEL, D. Introduction to soil physics. San Diego: Academic, 1982. 365 p.

HOLTZ, R. D.; KOVACS, W. D. An introduction to geotechnical engineering. Englewood Cliffs: Prentice-Hall, 1981. 733 p. 
HORN, R.; LEBERT, M. Soil compactability and compressibility. In: SOANE, B. D.; OUWERKERT, C. van (Ed.). Soil compaction in crop production. Amsterdam: Elsevier, 1994. p. 45-69.

IMHOFF, S.; SILVA, A. P.; DIAS JUNIOR, M. S.; TORMENA, C. A. Quantificação de pressões críticas para o crescimento das plantas. Revista Brasileira de Ciência do Solo, Viçosa, MG, v. 25, p. 11-18, 2001.

KEMPER, W. D.; ROSENAU, R. C. Aggregate stability and size distribution. In: KLUTE, A. (Ed.). Methods of soil analysis. Madison: American Society of Agronomy, 1986. part 1, p. 425-442. (Agronomy, 9).

KIEHL, E. J. Manual de edafologia. São Paulo: Ceres, 1979. $262 \mathrm{p}$.

KLUTE, A. Laboratory measurement of hydraulic conductivity of saturated soils. Madison: American Society of Agronomy, 1986. p. 253-261 (Monograph, 9).

KONDO, M. K.; DIAS JUNIOR, M. S. Compressibilidade de três latossolos em função da umidade e uso. Revista Brasileira de Ciência do Solo, Viçosa, MG, v. 23, p. 211-218, 1999.

LARSON, W. E.; GUPTA, S. C. Estimating critical stress in unsaturated soils from changes in pore water pressure during confined compression. Soil Science Society of America Journal, Madison, v. 44, n. 6, p. 1127-1132, 1980.

LEBERT, M.; HORN, R. A method to predict the mechanical strength of agricultural soils. Soil and Tillage Research, Amsterdam, v. 19, p. 275-286, 1991.

OLIVEIRA, L. B. de. Determinação da macro e microporosidade pela mesa de tensão em amostras de solo com estrutura indeformada. Pesquisa Agropecuária Brasileira, Rio de Janeiro, v. 3, p. 197-200, jan./fev. 1968.

PREVEDELLO, C. L. Física do solo. Curitiba: C. L. Prevedello, 1996. 446 p.

SANTOS, M. N. Influência de diferentes sistemas de manejo nos teores de carbono orgânico e nutrientes e no tamanho e distribuição de poros em um Latossolo Vermelho Escuro Argiloso na Região dos Cerrados. 1997. 133 f. Dissertação (Mestrado em Agronomia) - Universidade de Brasília, Brasília, 1997.

SILVA, A. P.; KAY, B. D.; PERFECT, E. Characterization of the least limiting water range of soils. Soil Science Society of America Journal, Madison, v. 58, p. 1775-1781, 1994.

SILVA, V. R.; REINERT, D. J.; REICHERT, J. M. Susceptibilidade à compactação de um Latossolo Vermelho-Escuro e de um Podzólico Vermelho-Amarelo. Revista Brasileira de Ciência do Solo, Viçosa, MG, v. 4, p. 239-249, 2000.

SNEDECOR, G. W.; COCHRAN, W. G. Statistical methods. Ames: Iowa State University Press, 1989.503 p.

SOANE, B. D. The role of organic matter in soil compactability: a review of some practical aspects. Soil and Tillage Research. Amsterdam, v. 16, p. 179-201, 1990.

SOWERS, G. F. Consistency. In: BLACK, C. (Ed.). Methods of soils analysis. Madison: American Society of Agronomy, 1965. p. 391-399.

TORMENA, C. A.; ROLOFF, G.; SÁ, J. C. Propriedades físicas do solo sob plantio direto influenciadas por calagem, preparo inicial e tráfego. Revista Brasileira de Ciência do Solo, Viçosa, MG, v. 22, p. 301-309, 1998. 